\title{
Biología y filosofía: en torno al naturalismo de John Dewey
}

\author{
Biology and philosophy: \\ on John Dewey's naturalism
}

\author{
JAIME FISHER \\ Instituto de Filosofía, Universidad Veracruzana (México)
}

Recibido: 15-1-2012

Aprobado definitivamente: 19-2-2013

\section{RESUMEN}

La principal influencia de la biología darwinista sobre la filosofía es el naturalismo. Tal ascendencia se refleja de manera muy clara en y a través de la obra de John Dewey, para quien la relación entre naturaleza y experiencia -o cultura-, fue siempre el problema preponderante de la filosofía. Desde un punto de vista historiográfico se sostiene que Dewey es el primer filósofo en tomarse en serio a Darwin. Aunque afirmar esto quizá no resulte muy arriesgado -sobre todo entre filósofos de la biología-, se pretende sirva como contexto para hacer volver la mirada hacia el pragmatismo, y en particular al experimentalismo como método filosófico de gran fertilidad, relevancia y actualidad. Intento articular esto siguiendo los conceptos relacionados de evolución, cognición y cultura.

\section{PALABRAS CLAVE}

\section{TRANSACCIÓN, EXPERIMENTALISMO, EVOLUCIÓN, COGNICIÓN, CULTURA}

\section{ABSTRACT.}

The chief influence of Darwinian biology on philosophy is naturalism. This impact is clearly manifest in and through John Dewey's work, wherein the relationship between nature and experience - or culture- is the outstanding problem for philosophy. So, from a historiographical point of view it is claimed that Dewey is the first philosopher to take Darwin seriously. Although 
this is not a risky claim to make -especially among philosophers of biology-, what I want to do here is redirect reader's attention to pragmatism, and particularly to experimentalism, as a philosophical method of great fertility, relevance and contemporary application. I try to formulate all this in terms of evolution, cognition and culture as related concepts.

KEY WORDS

TRANSACTION, EXPERIMENTALISM, EVOLUTION, COGNITION, CULTURE

Sin duda, el mayor disolvente de añejas preguntas en el pensamiento contemporáneo, el mayor desencadenante de nuevos métodos, nuevas intenciones y nuevos problemas, fue el llevado a cabo por la revolución científica que halló su culminación en El origen de las especies.

John Dewey: «The Influence of Darwin on Philosophy»

Para Dewey la teoría darwinista exigía en su momento -y creo que hoy lo exige de forma más urgente- efectuar un cambio no sólo en el contenido de nuestros problemas, sino en las maneras de formularlos y buscarles solución. Desde su perspectiva la relación entre la naturaleza física y biológica, por un lado, y la naturaleza humana o cultura, por otro -relación que no es otra que la existente entre el cuerpo y la mente-, se constituye no sólo como un problema sino más bien como el problema con el que la filosofía ha estado lidiando, en algunas ocasiones con menos fortuna que en otras.

La teoría de la evolución por selección natural hizo a Dewey percibir la necesidad de una reconstrucción en y de la filosofía o, como quiero proponer siguiéndolo, la necesidad de una adaptación permanente de tal filosofía al medio ambiente simbólico-cultural. De manera más exacta, la adecuación permanente del método filosófico, a saber, el experimentalismo, constituiría la manera operativa más inteligente al alcance del ser humano para el ajuste del proceso civilizatorio a los cambios medioambientales por él mismo producidos, y padecidos o disfrutados, según el caso. La filosofía de Dewey trazaría y recorrería, así, el camino desde la biología hasta la cultura (ciencia, tecnología, religión, arte, política) en la investigación de los instrumentos teórico-prácticos más eficientes para responder individual y colectivamente a la pregunta perenne acerca de cómo vivir.

En muy apretada síntesis, puede decirse que si la mente es una propiedad que ha emergido de formas de vida previas carentes de ella, y en tanto tal propiedad ha permitido la supervivencia $y$, al menos en ocasiones la bienvivencia 
también de al menos una parte de la especie, entonces esa propiedad puede y debe generar una explicación fiable de lo que son el conocimiento, la moralidad y la política; es decir, tendrá la finalidad central de formular una guía de la acción adaptativa. En este sentido concreto, el naturalismo de impronta darwinista y evolucionista es, antes que nada y en su médula, un proyecto de naturalizar la razón y, a través de ello, de llevar a cabo una crítica profunda y permanente de toda práctica humana intencional, científica, tecnológica, artística, política, y, en general, cultural. En tal sentido el experimentalismo puede ser visto y entendido como un intento de perseguir los mismos fines de la Ilustración a través de revaluar y reformular sus medios.

Es entonces posible afirmar que, mediante su ascendiente sobre Dewey, la principal influencia de Darwin sobre la filosofía consiste -de manera análoga y complementaria al paso del mito al logos operado por los primeros filósofos jonios-, en el tránsito de la Razón a la razón, es decir, en concebir a ésta ya no como una cualidad o potencia, lógica y cronológicamente previa a la práctica humana en un mundo independiente y escindido de ella, mundo que entonces sería susceptible de ser contemplado y aprendido de manera absoluta y con certeza, como querría Platón; sino más bien entenderla como un resultado evolutivo y transaccional, impulsado y alcanzado por la necesidad de conducir una determinada práctica orgánica habitual en dicho mundo, práctica que -dado el flujo heraclíteo al que está sometido ese mundo de fenómenos y cambios que de manera irremediable ocurren 'dentro de la caverna'-, requiere revisar de manera permanente sus condiciones y resultados y, en su caso, ajustar las primeras en tanto medios para corregir los segundos en tanto fines; donde medios y fines no pueden tampoco escindirse ontológicamente de tal práctica.

$S i$ la razón misma ha evolucionado junto al ser humano, entonces tiene un valor y una función adaptativa. Para Dewey esto no podía significar otra cosa que rechazar el dualismo ontológico que se suponía existente entre teoría y práctica, y esto como una consecuencia lógica y directa del rechazo de la biología evolucionista contra el dualismo organismo-medio ambiente.

Naturalismo se dice de muchas formas, y es un concepto sujeto a debates en los que por motivos obvios no me detendré. Por naturalismo entiendo aquí tres cosas vinculadas entre sí: 1) que la ontología se agota en la naturaleza y, por tanto, que la ciencia en general y las ciencias vinculadas a la biología en particular, son capaces de producir conocimiento suficiente, aunque falible, respecto al mobiliario del mundo; incluyendo ciencia y filosofía entre tal mobiliario; 2) que hay un continuum entre naturaleza y cultura en el sentido de que los fenómenos culturales - posibilitados por la complejidad evolutiva del sistema nervioso- son un subconjunto lógico de los fenómenos naturales; y 3), derivado de lo anterior, y existiendo también un continuum entre ciencia y filosofía, que esta última no puede (o no debería) llevar a cabo sus especulaciones 
ni diseñar sus métodos al margen, ni mucho menos en contra del conocimiento científico. En este sentido la naturalización de la razón, de manera contrastante con la concepción esencialista antigua del logos y de la res cogitans cartesiana, es el proyecto de llevar a sus últimas consecuencias la recursividad humana: la capacidad de pensarse a sí mismo como una especie más en la naturaleza, y la capacidad del cerebro para reflexionar sobre sus funciones, en particular las relacionadas con la intencionalidad de la acción.

Dewey afirmó, en su vena muy optimista, que «la influencia de Darwin sobre la filosofía estriba en haber conquistado los fenómenos de lo vivo para el principio de transformación y, con ello, haber liberado a la nueva lógica para su aplicación a los fenómenos de la mente, la moral y la vida propiamente humana». ${ }^{1}$ Me parece que esto no ha sido así, aunque, por supuesto, así debería haber ocurrido ya. Los seres humanos, incluidos muchos filósofos, parecen obsesionados aún con ideas de lo fijo, lo inmutable, lo perfecto y lo eterno, es decir, con ideas supernaturalistas. Muchos otros, como espejo de esta actitud, y como reacción instintiva ante la falibilidad natural de la razón, han adoptado ideas relativistas según el conocido, extendido y abominable principio posmoderno del todo vale. Ninguno de los dos extremos es razonable ni aceptable, y el naturalismo, sin ser en forma alguna un 'término medio', parece la manera más adecuada de evitar ambos.

En cualquier caso, un hecho cuya reconstrucción historiográfica está aún por hacerse, es que John Dewey, un filósofo estadounidense con un pasado intelectual profundo enraizado en Hegel, se empeñó con entusiasmo y optimismo en obtener todas las consecuencias filosóficas, $i$. e., prácticas, de $E l$ origen de las especies, texto que resultó en su momento un título y, sobre todo, un argumento corrosivo y disolvente de los viejos problemas, concepciones y métodos filosóficos, y, en particular, de la tarea y preocupación por la búsqueda de esencias, formas, cosas en sí u otras entidades no accesibles de forma directa ni indirecta, bien a la razón o bien a la experiencia. Dewey rechazará por ello la búsqueda de la certeza, estableciendo ya un consistente programa de naturalización en la epistemología. ${ }^{2}$ Pero bajo ninguna interpretación posible la obra de Darwin podía ni puede ser entendida como un disolvente de la razón misma, sino como la manera de entenderla entre el mobiliario del mundo

1 The Influence of Darwin on Philosophy

2 The Quest for Certainty. Ha de añadirse que no se refiere sólo a la llamada 'carga teórica de la observación', sino, lo que es más importante aquí, con lo que podemos llamar la 'carga biológica de la cognición': no sólo vemos y conocemos el mundo a partir de nuestros conocimientos previos y nuestras expectativas, sino que lo percibimos -de más radical maneraa través de un sistema nervioso cuyas características han evolucionado en la transacción entre organismo y medioambiente. 
biocultural propio del ser humano; y de manera más clara, como la conexión entre naturaleza y cultura. ${ }^{3}$

Dewey rechaza por ello las preocupaciones metafísicas en ética y epistemología, y, siguiendo a Peirce junto a Darwin, se inclinará por un falibilismo que caracteriza al conjunto de su obra en lógica, epistemología, filosofía moral y política, y que dará sentido a su experimentalismo; ${ }^{4}$ un método filosófico que, según mi punto de vista $-\mathrm{y}$ aquí sí arriesgándome un poco más-, puede ser visto como el análogo intencional, al tiempo que la alternativa cultural, a los azarosos tanteos del 'relojero ciego' que operan en el mundo biológico. En otras palabras, en la filosofía y en la cultura sí es posible un diseñador, o, si se quiere seguir la metáfora de Paley retomada por Dawkins, de un relojero, y, además, es posible que - contra Dawkins-, tal relojero no sea ciego, y también -en favor de Paley-, que sí sea inteligente. Lo que intenta Dewey es sintonizar la filosofía-como crítica de la cultura-con los descubrimientos de las ciencias y con los requerimientos prácticos, morales y políticos que impone el desenvolvimiento científico y tecnológico. Esto hará de su obra una filosofía moral y política preocupada en lo fundamental por la libertad. ${ }^{5}$

De acuerdo con su programa habría que ajustar a la cultura, es decir, «ese todo complejo que incluye conocimientos, creencias, arte, moral, derecho, costumbres, y cualesquier otras capacidades o hábitos adquiridos por el hombre en tanto miembro de la sociedad $»,{ }^{6}$ con los nuevos descubrimientos científicos; y de ahí que muchos años después, frente al proceso civilizatorio que había producido ya la Primera Guerra Mundial y que estaba produciendo la mayor crisis económica hasta ese momento, escriba: «Las máquinas significan un potencial de poder insospechado. Si hemos orientado este poder al dólar y no a la liberación y enriquecimiento de la vida humana es porque nos

3 Contra Descartes y Kant, Dewey sostendrá, parado sobre los hombros de Darwin, que el «alma» del primero y la «mente» del segundo se encuentran al alcance de la investigación empírica, es decir, que sí son fenómenos en principio explicables por la ciencia; y de ahí que la autoridad del entendimiento humano radique más en la capacidad de experimentar e intervenir en la naturaleza que en una observación contemplativa en el sentido de pasiva, introspectiva o trascendental.

4 Desde un punto de vista lógico y práctico el experimentalismo es una consecuencia del falibilismo: si nunca falláramos en las creencias que guían nuestra práctica no sería necesario experimentar para corregirlas.

5 The Public and its Problems, Individualism Old and New, Liberalism and Social Action.

6 Tylor, Edward B. (1920 [1871]): Primitive Culture, Sixth Edition, John Murray, London. Dewey retoma esta definición canónica y amplia en antropología para apuntalar su idea de experiencia como transacción en y desde una cultura, cosa ésta que sería, a su vez, el (los) objeto(s) de la crítica filosófica (ciencia, arte, moral, derecho, hábitos, etc.) Véase Experience and Nature, en especial el capítulo II. 
hemos conformado con no exceder los límites de los objetivos y valores tradicionales a pesar de estar en posesión de un instrumento de transformación revolucionario». ${ }^{7}$

Lo que Dewey el reformador social se propone y persigue es la transmutación de los valores morales y políticos a partir de los valores asociados al desenvolvimiento de la ciencia (verdad, objetividad, fertilidad, publicidad, razonabilidad, corregibilidad, etc. $\left.{ }^{8}\right) \mathrm{Si}$ la especie evoluciona de manera aleatoria, pero la cultura lo hace de manera intencional, entonces los valores que guían toda práctica cultural deberán evolucionar adecuándose a los cambios introducidos en el mundo por la acción humana. Por esto afirma que "la tarea característica, los problemas y la materia de la filosofía surgen de las presiones y reacciones que se originan en la vida de la comunidad misma en que surge una filosofía determinada [esto es, en el medio ambiente biocultural humano] y que, por tal razón, los problemas especificos de la filosofía varían [es decir, 'la filosofía se adapta'] en consonancia con los cambios que se producen constantemente en la vida humana. ${ }^{9}$ De acuerdo con esto la filosofía debería ser una crítica de la cultura; y, siendo la primera una parte de la segunda, la filosofía sería entonces una crítica de sí misma, crítica que desembocaría en un ajuste o adaptación permanente entre filosofía y experiencia científica, moral y política, o, como propondrá Dewey casi al final de su vida y ante diversas objeciones (entre las que destacan las de Bertrand Russell), un ajuste permanente entre filosofía y cultura.

Conviene en este recodo del camino, una vez que hemos topado con Bertrand Russell y su persistente crítica y rechazo del pragmatismo por su presuntos vínculos con el capitalismo estadounidense, citar un comentario del propio Dewey al respecto: «[...] creo que el hábito, persistente en Russell, de vincular la teoría pragmatista del conocimiento con los aspectos más repugnantes del industrialismo estadounidense, en lugar de hacerlo con el método experimental de producir conocimiento, es tanto como si yo vinculara su filosofía [la de Russell] con los intereses de la aristocracia terrateniente inglesa en lugar de hacerlo con su predominante interés [el de Russell] en las matemáticas»». ${ }^{10} \mathrm{En}$ el mismo texto Dewey pondrá en claro y señalará - de la manera más cortés que estuvo a su alcance- más de un non sequitur en la argumentación antipragmatista de un lógico tan prestigiado como fue y es Russell.

Lo que interesa de esto es que varias de las polémicas en que ambos participaron tuvieron su origen básico en el presunto hiato entre lo material y lo

$7 \quad$ Individualism Old and New; LW, Vol. 5, p. 87.

8 Theory of Valuation, LW, Vol. 13

9 Reconstruction in Philosophy, MW; Vol. 12, p. 256.

10 «Experience, Knowledge and Value: A Rejoinder», LW, Vol. 14, p. 13. 
espiritual, entre cuerpo y mente o, de forma más explícita, entre naturaleza y cultura; dualismos heredados de la filosofía antigua y que no podían, en opinión de nuestro autor, mantenerse en pie tras la publicación de El origen de las especies. Meter a Russell en este ensayo entonces cobra pertinencia no sólo por su talla filosófica e intelectual, ni tampoco tiene que ver sólo con su oposición pertinaz al pragmatismo, sino con su negativa expresa de adjudicar a la teoría de la evolución relevancia filosófica alguna, ${ }^{11}$ cosa que constituye el polo opuesto al sostenido por Dewey.

El pragmatismo en general, y por supuesto también la versión experimentalista desarrollada por él, pone en el centro de la reflexión filosófica a la acción humana intencional y considera sus condiciones y resultados, cosas que conducen a y presuponen cierto uso de la razón. Las culturas en que se manifiestan esas acciones significarían distintas formas técnicas y valorativas para mejorar la vida de quienes participan en ellas, es decir, serían distintas expresiones de la razón para responder a la pregunta sobre cómo vivir.

No parece por ello muy aventurado afirmar aquí que la obra de Dewey es una teoría filosófica de la evolución de la especie humana a través de la selección cultural, donde en contraste y continuidad con la biología, tanto el mecanismo de variación -la acción humana que inaugura y/o cancela posibilidades y caminos para la sobrevivencia y la bienvivencia-, como el de selección -la sedimentación de esas acciones en la o en las culturas-son intencionales. ${ }^{12}$

Un hecho sobresaliente, y en algún sentido problemático en la práctica filosófica de John Dewey, es lo fragmentario de su obra; al grado que él mismo sólo muy tarde reconoció que en su trabajo sí era discernible -y defendibleun sistema filosófico consistente. ${ }^{13}$ Vista su vasta obra a ojo de pájaro da la impresión de haber sentido la necesidad y el impulso de comenzar por todas partes a la vez; y parece que todo lo que pudo hacer fue 'vencer la tentación sucumbiendo de lleno en sus brazos', pues desde muy temprano ataca problemas diversos que de manera simultánea le acompañarán durante toda su prolífica vida. La sistemicidad de su filosofía es una consecuencia no intencional de

11 «What biology has rendered probable is that the diverse species arose by adaptation from a less differentiated ancestry. This fact is in itself exceedingly interesting, but it is not the kind of fact from which philosophical consequences follow», Bertran Russell, citado por Cunningham (1996), p. 1.

12 De aquí se seguiría una tesis contra el multiculturalismo normativo -por lo demás consistente con su liberalismo-, pues implica que no todas las culturas valdrían lo mismo o tendrían el mismo valor y que, de hecho, el ejercicio de la inteligencia humana en evaluar esas culturas como mecanismos de selección sobre las eventuales variaciones introducidas por la técnica, considerada ésta en sentido amplio como acción intencional sistemática.

13 «Nature in Experience», The Philosophical Review, Vol. 49, No. 2 (Mar., 1940), pp. 244-258. 
su práctica, una especie de propiedad emergente de su ensayística. Y es que así como El ensayo sobre el principio de la población de Malthus influyó a Darwin, la obra de éste impactó en Dewey. De la misma manera en que la teoría de la evolución revolucionó la biología, la obra de Dewey -siguiendo esas ideas- reconoce la necesidad de una reconstrucción de y en la filosofía, y se avocó de forma inmediata a ello y en todas las direcciones que le pareció necesario, de una manera más bien anárquica y sin un plan preconcebido para construir un sistema.

La teoría de la evolución -en manos de Dewey-se convierte en un revulsivo de toda filosofía (incluyendo por supuesto la suya propia) en el sentido preciso de hacerla en relación a los resultados de las ciencias, y, por tanto, en el impulso naturalista para considerar al hombre y sus productos -incluyendo a la filosofía misma, y con ella a esa vieja señorita llamada Razón- como objetos susceptibles de estudiarse y conocerse de la misma manera en que llevamos a cabo el estudio y conocimiento de otras especies, y del resto de la naturaleza.

La primera referencia escrita de Dewey a la teoría de la evolución data de $1883 .{ }^{14}$ Ahí endereza su crítica contra toda idea de absoluto, oponiéndole la idea evolucionista de relación situacional entre organismo y medio. Aquí mismo también tiene origen su concepto de transacción, que constituye base y eje de su ontología antiesencialista, cimiento que servirá al posterior desarrollo de una epistemología y una axiología naturalistas, y que, pese al ya señalado carácter disperso de su obra, dará consistencia a su sistema filosófico. La idea central es que tanto en el mobiliario del mundo físico, como en el biológico y el simbólico-cultural, nunca encontramos 'entidades', 'cosas en sí', ni 'esencias', sino sólo transacciones. El concepto apunta contra todo tipo de dualismos y, en y desde el mundo biológico en particular, contra la dicotomía entre organismo y medio ambiente. Por transacción se entiende el complejo de condiciones situacionales en que se producen de forma simultánea tanto el medio ambiente como el organismo. Su referencia empírica está constituida por dos hechos entrelazados: 1) que ambiente $\boldsymbol{y}$ organismo emergen en el mismo vínculo fundacional material: que no pueden existir-en un sentido ontológico-, uno al margen de su transacción con el otro; y 2) que en sus sentidos lógico y cronológico no pueden existir antes de esa transacción, misma que entonces expresa una relacionalidad básica: el vínculo funcional en que ambos, organismo $y$ ambiente, se fundan y originan.

Sobre esta concepción de clara impronta darwinista sostendrá su método experimentalista. Escribirá de manera autobiográfica que su acercamiento a la biología -a través de un curso tomado a fines de la década de 1870, en el que el texto básico fue la Fisiología de Thomas Huxley ('the Darwin's Bulldog')-, le 
generó 'un sentido de interdependencia y unidad interrelacionada que dieron forma a [sus] primeros tanteos intelectuales, creando una forma de ver las cosas al que debía adaptarse cualquier campo de interés, ${ }^{15}$ incluyendo no sólo a la epistemología sino también a la moral. ${ }^{16}$ Tal sentido de interdependencia y unidad interrelacionada -al que adaptó todo su interés -, constituye su concepto de transacción.

Aplicará la idea de manera clara y eficaz en su crítica al concepto de arco reflejo en psicología,${ }^{17}$ donde rechaza la dicotomía entre estímulo y respuesta, y entiende los procesos cognitivos precisamente como una coordinación organismo-medio ambiente. La cognición tendrá entonces una función adaptativa en el sentido de la supervivencia del organismo, proceso que para todo caso ocurre, más que en el medio ambiente, a través de éste. En el caso del ser humano la cultura aparece también como una 'segunda naturaleza' que le permitirá no sólo sobrevivir, sino incluso bienvivir, o por lo menos intentarlo. Y aquí es donde la naturalización de la razón cobra importancia como el vínculo entre biología y cultura, o, o como Dewey prefirió ponerlo, entre naturaleza y experiencia.

A través de esta idea clave remarca el papel del medio ambiente físico y simbólico cultural, tanto en la formulación de los problemas humanos como en sus posibles vías de solución. En filosofía -siguiendo a James-, sostendrá que las ideas y métodos de ésta deberán adaptarse al medio, y que la prueba de su adaptación o inadaptación, es decir, de su selección positiva o negativa, será si funcionan a no lo hacen al conducir una práctica determinada. Hacer y padecer, y en su caso corregir el hacer para evitar el padecer, es la particular transacción mayor que Dewey denomina experiencia, y casi al final de su vida, cultura, entendida como proceso intencional de autocultivo, individual y colectivo. Esta ocurre en la naturaleza, a través de la naturaleza y es de la naturaleza, tanto en el sentido en que su objeto es una parte del medio ambiente natural, como en el sentido de que su sujeto es también un organismo natural. En un momento esto lo llevó a definir su método como empirismo naturalista y también como naturalismo empirista. ${ }^{18}$

15 «rom Absolutism to Experimentalism»; LW, Vol. 5

16 «Evolution and Ethics», de 1898 (EW Vol. 5), «The Evolutionary Method as Apllied to Morality», de 1902 (MW, Vol. 2) y «Logical Conditions of a Scientific Treatment of Morality» de 1903 (MW, Vol 3), son tres ensayos cuyo tema será complementado y ampliado en Logic: Theory of Inquiry, de 1938 (LW Vol. 12), y en Theory of Valuation, de 1939 (LW Vol.13); todo en plena consistencia con la teoría evolucionista y el rechazo al dualismo organismo-medio ambiente

17 «The Reflex Arch Concept in Psychology»; EW, Vol. 5

18 Nature and Experience. De manera desafortunada en otras ocasiones definió su método como instrumentalismo, cosa que, además de generar diversos malos entendidos y confusiones, 
Aunque su preocupación por la filosofía moral y política está presente desde muy temprano en su obra, ${ }^{19}$ no sería sino hasta después de escribir Experience and Nature que dedicará de forma más consistente su trabajo a las implicaciones del experimentalismo en esos temas. En The Public and its Problems $^{20}$ rechaza la dicotomía individuo-sociedad al tiempo que establece criterios empíricos claros para una distinción política estricta entre lo público y lo privado, considerando para ello la transacción medioambiental y, en este caso, la transacción cultural propia de las sociedades humanas, definiendo de paso tanto las funciones como el origen empírico y transaccional del Estado. Profundiza su filosofía política en Individualism, Old and New, donde critica la cultura de su momento (1929) sosteniendo que «la individualidad es inexpugnable debido a que es una forma particular de sensibilidad, selección, elección, respuesta y aprovechamiento de las condiciones medioambientales [...]»; pero que, al mismo tiempo, la cultura de masas está conduciendo a la pérdida del individuo y a su total desorientación moral y política. Liberalism and Social Action ${ }^{21}$ puede considerarse como la culminación de su reflexión política, planteando que el crecimiento de la libertad del individuo ha de ser conseguida por medios de la acción social, es decir, que el grupo, la sociedad, y el Estado en particular, son medios para el crecimiento de la libertad individual, y que, como cualesquier otros medios o instrumentos, han de ser evaluados en su aceptabilidad racional o legitimidad según su eficacia en alcanzar tal fin.

Vinculada de manera muy estrecha con su filosofía política se encuentra su teoría de la educación antes desarrollada en Democracy and Education, ${ }^{22}$ en donde traza el vínculo entre la educación y la libertad en el sentido indicado líneas arriba, sentido que no sólo se distingue sino que incluso se contrapone al concepto típico del liberalismo económico de laissez-faire, y del cual, pese a todo, suelen Dewey y los pragmatistas en general ser acusados de manera constante.

Durante toda su producción intelectual vinculará sus reflexiones filosóficas en epistemología con la cognición como un fenómeno transaccional de adaptación biológica entre organismo y medio. En una serie de artículos escritos junto a Arthur Bentley, y que aparecen publicados en forma de libro a mitad

sirvió de base a sus críticos para acusarle de defender una racionalidad meramente instrumental, y de intentar hacer ver su filosofía como un mero epifenómeno de la 'cultura estadounidense', guiada por la acumulación capitalista.

19 «Moral Theory and Practice» (1891); «The Philosophy of Thomas Hill Green» (1889); EW Vol. 3

20 LW, Vol. 2.

21 LW, Vol. 11.

22 MW, Vol.9 
del siglo pasado, ${ }^{23}$ el concepto de transacción y sus bases biológico-evolutivas terminarán teniendo su expresión más acabada y completa.

Los procesos cognitivos y su producto emergen por necesidad desde una situación problemática y situacional-transaccional mayor en la que organismo $\boldsymbol{y}$ medio ambiente se hallan involucrados. Característico del hombre es plantearse problemas; pero, rescatando a Peirce, no cualquier cosa es un problema: los problemas acosan al hombre por todas partes sólo en virtud de su transacción con un ambiente en el que no ha sido puesto artificial ni de manera intencional, sino desde y a través del que ha emergido naturalmente, por mero azar, evolución y selección natural. No puede el hombre entonces plantearse problemas en ciencia o en filosofía que no sean problemas referidos a esa transacción situacional, que es a lo que se refiere el propio Peirce cuando sugiere que no podemos dudar en filosofía lo que no dudamos en nuestro corazón. La investigación, científica o del sentido común, sólo se dispara a partir de un problema, de una irritación práctica que requiere y urge al ser humano a tomar un curso de acción y evitar otros. Esto implica la libertad, la intencionalidad y la racionalidad de la acción humana.

Es en este sentido que para nuestro autor nada habrá más práctico que una buena teoría filosófica. Pensó siempre al pragmatismo, y a su versión experimentalista, como parte de un movimiento más amplio y general de permanente reconstrucción intelectual y civilizatoria cuyo impulso inicial habría sido $E l$ origen de las especies; una constante reconstrucción no sólo en las ciencias, sino también en la filosofía, la moral, y la política. Un proyecto, en todo caso, no sólo para sobrevivir, sino para bienvivir, es decir, para ese crecimiento de la libertad humana. La cultura (como cultivo a través de la experiencia) aparece en Dewey como la respuesta a la pregunta socrática. Su propuesta de reconstrucción consistirá en que tal crítica reconstructiva -que ha de ser llevada a cabo por la filosofía misma-, comience por los objetos y métodos de ésta, a imagen y semejanza de los objetos y métodos de la ciencia.

La idea seminal darwinista de transacción constituye el eje a través del cual se edifica el experimentalismo como versión naturalista del pragmatismo, permitiendo afirmar una influencia nodal de la biología sobre la filosofía, y de manera particular en la influencia de Darwin sobre el pensamiento de Dewey, cosa que animaría a explorar y ampliar esa influencia sobre el resto de la filosofía en su necesidad de permanente reconstrucción. Un camino a explorar bien puede ser considerar a la filosofía en su conjunto, y en particular a la filosofía política, como guía de una ingeniería social (ingeniería constitucional diría Sartori) capaz de sintonizar a la civilización mundial -y las diversas formas culturales en ella manifiestas-con los instrumentos que pone a nuestro alcance el desenvolvimien- 
to científico y tecnológico. Huelga decir que ese objetivo, sin embargo, rebasa a la filosofía, y sería más bien una tarea para la(s) ciudadanía(s).

\section{BiBLIOGRAFÍA}

CUNNINGHAM, S. 1996: Philosophy and the Darwinian Legacy, University of Rochester Press.

HICKMAN, L. 2003: The Collected Works of John Dewey, 1882-1953. Electronic edition, InteLex Corporation, Charlottesville, Virginia.

POPP, J. A. 2007: Evolution's First Philosopher: John Dewey and the Continuity of Nature, State University of New York Press, Albany.

TONONI, G. 2012: Phi: A Voyage from the Brain to the Soul, Pantheon Books, New York.

JaIME FisHer es Investigador de tiempo completo en el Instituto de Filosofía de la Universidad Veracruzana, Xalapa (México)

Lineas de investigación:

Filosofía de la ciencia, filosofía política y filosofía de la cultura

Publicaciones recientes:

(2011) El hombre y la técnica: hacia una filosofía política de la ciencia y la tecnología; UNAM, México; ISBN 978-607-02-1848-4

(2012) «Problemas del Multiculturalismo», en Ética, política y responsabilidad: reflexiones interdisciplinares, Uniminuto, Bogotá.ISBN 978-958-763-064-0

Dirección electrónica: jaime.fisher@gmail.com o jfisher@uv.mx 